\title{
TRANSEXUALIDADE, ORDEM MÉDICA E POLÍTICA DE SAÚDE: CONTROLE NORMATIVO DO PROCESSO TRANSEXUALIZADOR NO BRASIL
}

\author{
Manoel Antônio dos Santos \\ Faculdade de Filosofia, Ciências e Letras de Ribeirão Preto, Universidade de São Paulo \\ Ricardo Santos de Souza \\ Faculdade de Medicina de Ribeirão Preto, Universidade de São Paulo \\ Lúcia Alves da Silva Lara \\ Faculdade de Medicina de Ribeirão Preto, Universidade de São Paulo
}

Eduardo Name Risk

Faculdade de Filosofia, Ciências e Letras de Ribeirão Preto, Universidade de São Paulo

Wanderlei Abadio de Oliveira

Escola de Enfermagem de Ribeirão Preto, Universidade de São Paulo

Vinicius Alexandre

Faculdade de Filosofia, Ciências e Letras de Ribeirão Preto, Universidade de São Paulo

Érika Arantes de Oliveira-Cardoso

Faculdade de Filosofia, Ciências e Letras de Ribeirão Preto, Universidade de São Paulo

\section{Resumo}

Este estudo teve por objetivo examinar a trajetória histórica do posicionamento oficial do Conselho Federal de Medicina (CFM) acerca dos procedimentos médicos associados às demandas do Processo Transexualizador (PrTr). Como objetivo específico, discutir as implicações das normatizações existentes, que regulam as intervenções médicas no campo da transgenitalização, para que se possa repensar a formação médica e de outros profissionais de saúde. O método utilizado consiste na análise documental, realizada a partir do banco de dados de pareceres, resoluções, notas técnicas, despachos e recomendações do CFM, com foco nos documentos oficiais de domínio público, que abordam o posicionamento dessa entidade representativa da classe médica em relação à realização de cirurgias de transgenitalização e prescrição da hormonioterapia para pessoas trans. Por meio dessa análise foi possível traçar, retrospectivamente, a evolução desse posicionamento e identificar quais foram os prováveis fatores que desencadearam progressivas mudanças da posição oficial assumida pelo CFM em relação à legalidade e aos aspectos éticos envolvidos nessas intervenções.

Palavras-chave: cirurgia de readequação sexual; pessoas transgênero; identidade de gênero; políticas públicas; saúde pública. 


\title{
TRANSEXUALITY, MEDICAL ORDER AND HEALTH POLICY: THE REGULATORY CONTROL OF THE TRANSSEXUALIZING PROCESS IN BRAZIL
}

\begin{abstract}
This study aimed to examine the historical trajectory of the official position of the Federal Medical Council (FMC) regarding medical procedures associated with the demands of the Transsexualizing Process (PrTr). As a specific objective, to discuss the implications of existing norms, which regulate medical interventions in the field of transgenitalization, in order to rethink the medical and other health professionals' training. The method used consists of the documentary analysis, based on the database of opinions, resolutions, technical notes, orders and recommendations of the FMC, focusing on official documents in the public domain, which deal with the positioning of this representative body of the medical profession in relation transgender surgery and prescription of hormone therapy for transgender people. Through this analysis, it was possible to trace, retrospectively, the evolution of this position and to identify which were the probable factors that triggered progressive changes of the official position assumed by the FMC in relation to the legality and the ethical aspects involved in these interventions.

Keywords: sex reassignment surgery; transgendered persons; gender identity; public policies; public health.
\end{abstract}

\section{TRANSEXUALIDAD, ORDEN MÉDICO Y POLÍTICA DE SALUD: EL CONTROL NORMATIVO DEL PROCESO TRANSEXUALIZADOR EN BRASIL}

\begin{abstract}
Resumen
Este estudio tuvo por objeto examinar la trayectoria histórica del posicionamiento oficial del Consejo Federal de Medicina (CFM) acerca de los procedimientos médicos asociados a las demandas del Proceso Transexualizador (PrTr). Como objetivo específico, discutir las implicaciones de las normativas existentes, que regulan las intervenciones médicas en el campo de la transgenitalización, para que se pueda repensar la formación médica y de otros profesionales de salud. El método utilizado consiste en el análisis documental, realizado a partir del banco de datos de opiniones, resoluciones, notas técnicas, despachos y recomendaciones del CFM, con foco en los documentos oficiales de dominio público, que abordan el posicionamiento de esa entidad representativa de la clase médica con relación a la realización de cirugías de transgenitalización y prescripción de la terapia hormonal para personas transgénero. Por medio de este análisis fue posible trazar retrospectivamente la evolución de ese posicionamiento e identificar cuáles fueron los probables factores que desencadenaron progresivos cambios de la posición oficial asumida por el CFM en relación a la legalidad ya los aspectos éticos involucrados en esas intervenciones.
\end{abstract}

Palabras clave: cirugía de reasignación de sexo; personas transgéneras; identidad de género; políticas públicas; salud pública.

\section{INTRODUÇÃO}

O Processo Transexualizador (PrTr) compreende um conjunto de estratégias assistenciais implantadas no Sistema Único de Saúde (SUS) em 2008 (Portaria no 457, de 19 de agosto de 2008, Brasil, 2008a) e regulamentadas e ampliadas em 2013 (Portaria no 2803, de 19 de novembro de 2013), direcionadas à atenção e cuidado integral de transexuais e travestis que tenham o desejo de realizar modificações corporais por meio da adequação da aparência física e da função das características sexuais, em conformidade com sua identidade de gênero. 
Por identidade de gênero entende-se o modo singular como uma pessoa define a si mesma enquanto alguém pertencente ao gênero masculino, feminino ou outro. Corresponde ao sentimento, fundado em uma convicção interna, de ser "homem", "mulher" ou qualquer outra definição.

Com a designação "pessoa transgênero" (ou, simplesmente, pessoa trans) entende-se aquela que tem identidade de gênero diversa da imposta pelos padrões binários (Popadiuk, Oliveira, \& Signorelli, 2017). Essa categoria inclui as travestis e transexuais. As pessoas trans subvertem a lógica dominante de que existe um alinhamento natural e inequívoco entre sexo biológico e gênero, ou seja, a crença no sexo de nascimento como determinante da identidade de gênero. Frequentemente, pessoas que escapam da lógica binária e dicotômica (homemmulher) estão sujeitas a sofrer preconceitos e discriminação.

A pessoa trans pode ou não passar por um processo de transgenitalização em determinado momento de sua vida, dependendo de seu desejo de readequar seu corpo e sua genitália ao gênero com o qual se identifica. O Processo Transexualizador (PrTr) é um procedimento pautado no modelo biomédico, regulamentado pelo Ministério da Saúde por meio da Portaria no 457, de 19 de agosto de 2008 (2008) e da Portaria no 2803, de 19 de novembro de 2013 (Portaria no 2803, de 19 de novembro de 2013). Essas portarias definem as diretrizes nacionais que regulam e normatizam o processo. Mediante a Portaria de 2013, o PrTr no âmbito do Sistema Único de Saúde (SUS) foi redefinido e ampliado.

Assim, desde 2008 a pessoa transexual tem assegurado seu direito à cirurgia de redesignação sexual no Sistema Único de Saúde (SUS). Segundo Popadiuk et al. (2017), o PrTr consiste em um conjunto de estratégias de atenção à saúde necessárias para a transformação dos caracteres sexuais. Esse processo de transgenitalização a que a pessoa transexual pode se submeter vem sendo implementado no âmbito do SUS pelo Ministério da Saúde com avanços e recuos. Nesse contexto é importante conhecer as normatizações médicas para a realização de hormonioterapia e cirurgias de transgenitalização em pessoas trans no cenário nacional. Esse conhecimento possibilitará contextualizar e compreender as barreiras encontradas no acesso aos serviços locais de saúde, especializados no PrTr. Para dimensionar corretamente essa questão, que ancora o presente estudo, é preciso situar e contextualizar o problema de pesquisa.

A saúde da população de lésbicas, gays, bissexuais, transexuais e travestis (LGBTT) tem constituído uma pauta relevante na arena da saúde na atualidade, 
com necessidades e especificidades que ainda se esforçam para ganhar visibilidade (Cardoso \& Ferro, 2012). Há uma crescente reivindicação de que o Estado se responsabilize e se aparelhe para responder às demandas da população LGBTT, reconhecida como vulnerável, mas também heterogênea e diversificada. Ao mesmo tempo, independentemente das questões relacionadas às orientações sexuais e identidades de gênero, nas últimas décadas a acessibilidade aos serviços de saúde passou a ser considerada como um componente crítico na determinação da qualidade de vida não apenas do indivíduo, mas de toda a sociedade (Silva, 2014).

Nesse contexto, a homo/transfobia pode constituir uma significativa barreira de acesso da comunidade LGBTT aos serviços de saúde, comprometendo a possibilidade de oferecimento de uma assistência de qualidade que contemple os pressupostos da integralidade. Com receio de ser alvo de preconceito e sofrer discriminação, a pessoa LGBTT, ao procurar os serviços de saúde, muitas vezes omitem sua orientação sexual, ou ainda o que é mais comum, têm sua sexualidade presumida pelos profissionais como sendo heterossexual, o que pode desencorajar o(a) paciente a aderir às orientações médicas (Silva, 2014). Isso requer a adoção de medidas que possam tornar o ambiente dos serviços de saúde mais acolhedor e receptivo a essa clientela que não se alinha à norma referendada na matriz binária heterossexual para os gêneros.

Considerando que a trajetória para efetivar a normatização médica do processo transgenitalizador no contexto brasileiro se estendeu por quase 40 anos, torna-se relevante realizar um levantamento retrospectivo que permita mapear a evolução dos posicionamentos do Conselho Federal de Medicina (CFM) em relação à temática abordada, identificando seus pressupostos e embasamento teórico, buscando compreender como e quando ocorreu a modificação desses posicionamentos e os possíveis determinantes de tais mudanças.

Este estudo teve por objetivo examinar a trajetória histórica da evolução do posicionamento oficial do CFM acerca dos procedimentos médicos associados às demandas do PrTr. O objetivo específico foi discutir as implicações das normatizações existentes, que regulam as intervenções médicas no campo da transgenitalização, para que se possa repensar a formação médica e de outros profissionais de saúde. 


\section{MÉTODO}

Estudo exploratório, tendo como base metodológica a proposta normativa de pesquisa de avaliação de programas e sistemas de saúde. O método utilizado consiste na análise documental, realizada a partir do banco de dados de pareceres, resoluções, notas técnicas, despachos e recomendações do CFM, com foco nos documentos oficiais de domínio público, que abordam o posicionamento dessa entidade representativa da classe médica em relação à prescrição da hormonioterapia e à realização de cirurgias de transgenitalização para pessoas transexuais.

\section{Instrumento}

Formulário para Extração de Informações Documentais

Foi elaborado um formulário para captação dos dados extraídos dos documentos consultados.

\section{Procedimento}

Por meio de busca em sites específicos foi realizada uma revisão dos pareceres, resoluções, notas técnicas, despachos e recomendações emitidos pelo CFM no período de 1975 a 2017.

O critério de inclusão adotado foi: documentos e normatizações que tinham como foco a cirurgia de transgenitalização e a hormonioterapia para pacientes transexuais, entendidas como recursos médicos para a transição de gênero.

O único critério de exclusão adotado foi: documentos cuja temática não se enquadrava ou não tinha como foco principal a questão da realização da cirurgia de transgenitalização e/ou prescrição da hormonioterapia para pessoas com transexuais.

Procedimento de coleta de dados e análise

O levantamento foi realizado no período de julho a agosto de 2017, mediante busca sistemática empreendida a partir dos termos: hormonioterapia, transexualidade, transexualismo, cirurgia de transgenitalização, cirurgia de transgenitalismo e cirurgia de conversão sexual, por meio do buscador disponível no site da médica, na página https://portal.cfm.org.br/index.php?option=com_normas.

Com uso do Formulário para Extração de Informações Documentais, o levantamento foi realizado de forma independente por dois pesquisadores, com 
experiência em coleta de dados em pesquisa documental. As convergências encontradas na avaliação dos dois examinadores foram consideradas. As divergências foram resolvidas por consenso entre eles.

Do material coligido foram descartados os documentos duplicados, sendo selecionados os mais relevantes com base na pergunta orientadora deste estudo: Como se deu o estabelecimento de normatizações médicas para a realização de cirurgias de transgenitalização e/ou hormonioterapia em pessoas transexuais no contexto nacional?

\section{RESULTADOS}

Foram encontrados 20 registros de documentos com o termo transexualismo, sete registros com o termo hormonioterapia, oito registros de transexualidade, 14 registros de cirurgia de transgenitalização, quatro registros de cirurgia de conversão sexual e 17 registros de cirurgia de transgenitalismo, totalizando 70 documentos. Esse número foi reduzido para 31 registros após descartar os documentos duplicados e, em seguida, para 14, quando removidos os materiais que não preenchiam o critério de inclusão, ou seja, cuja temática não se enquadrava na regulamentação da prescrição de hormonioterapia ou da cirurgia de transgenitalização em pessoas transexuais.

O primeiro pronunciamento do CFM em relação à abordagem de pacientes transexuais ocorreu por meio do Parecer-Consulta no 28/75 assinado pelo conselheiro Clarimesso Machado Arcuri, que se posicionou contra o procedimento, concluindo ser a cirurgia "não de mudança de sexo, pois muda-se a genitália e não o sexo do indivíduo, resultando em mutilação grave e ofensa à integridade corporal. O sexo biológico é imutável" (CFM, 1991). Tal ponto de vista se manteve em vigor nas décadas seguintes, conforme evidenciado pelo posicionamento do conselheiro Hilário Lourenço de Freitas Junior, membro do CFM que determinou, por meio do PC/CFM/No 11/1991, que: "Incorre em ilícito ético e penal o médico que, diante de solicitação de seu paciente, realizar cirurgia de conversão sexual, por desobservância ao artigo 129 do Código Penal e ao artigo 42 do Código de Ética Médica, por se tratar de mutilação grave e ofensa à integridade corporal". Resposta semelhante foi dada no Parecer-Consulta PC/CFM/No 12/1991, cujos objetivos eram semelhantes (CFM, 1991a; CFM, 1991b). 
A mudança no entendimento, que levaria a uma revisão do posicionamento que vigorou por décadas, parece ter se iniciado com o Parecer e Proposta de Resolução No 39/97, motivado, segundo argumentação do próprio CFM, pela preocupação com artigos "sobre cirurgia de transexualismo" publicados na imprensa leiga. O debate gerado pelo tema no final do século passado levou o CFM a criar uma comissão, formada pelos conselheiros Júlio Cézar Meirelles Gomes e Lúcio Mário da Cruz Bulhões, que concluíram que, apesar dos posicionamentos anteriores contrários ao procedimento médico, devido aos recentes estudos genéticos indicarem bases orgânicas para os desvios de identidade sexual, a presença de técnicas seguras e eficazes, além das mudanças de costumes e hábitos sociais dos grandes centros urbanos, optou-se pela liberação da cirurgia de transgenitalização, sob caráter experimental (CFM, 1997).

Esse parecer foi seguido nos anos posteriores pela resolução No 208, de 27/10/2009 do Conselho Regional de Medicina do Estado de São Paulo (CREMESP), que estabeleceu o direito dos pacientes ao uso do nome social, independentemente do nome, prenome do registro civil ou que consta no prontuário do serviço, bem como o direito a ter um atendimento baseado no respeito ao ser humano e na integralidade da atenção à saúde. Quatro anos mais tarde, o parecer No 8/13 do CFM autorizou e estabeleceu as diretrizes para realização da hormonioterapia em indivíduos transexuais, incluindo a inibição da puberdade em adolescentes transexuais (CREMESP, 2009; CFM, 2013).

\section{DISCUSSÃO}

Entre o primeiro posicionamento do CFM e a última manifestação oficial da entidade acerca dessa matéria houve um hiato de 38 anos. O primeiro pronunciamento oficial em relação ao processo cirúrgico de transgenitalização se deu por meio do Parecer-Consulta no 28/75, no qual o CFM se posicionava claramente pela ilegalidade do método. Decorridos 22 anos desse marco histórico deu-se finalmente a autorização desse procedimento, desde que em caráter experimental, mediante a publicação da Resolução no 1482 de 1997 . Dezesseis anos depois, em 2013, houve a liberação do uso da hormonioterapia em adolescentes transexuais, por meio do parecer no 8/13 (CFM, 1997, 2013).

Como apontado pelo próprio parecer No 39/97, parte da responsabilidade pela modificação do posicionamento do CFM em relação à legalidade dos 
procedimentos de cirurgia de transgenitalização e da hormonioterapia pode ser atribuída à influência da opinião pública a favor da legalização da realização do procedimento, diante da disseminação na imprensa leiga de informações sobre esse processo (CFM, 1997). Ainda de acordo com o mesmo parecer, os critérios diagnósticos adotados como pré-requisitos para a realização do procedimento foram baseados nas condutas estabelecidas pela Fundação Harry Benjamin (CFM, 1997), uma entidade dos Estados Unidos que é considerada referência na área.

No mesmo contexto, a decisão pela legalização da hormonioterapia, incluindo para adolescentes, foi motivada por uma combinação de fatores, a saber: a constatação do uso disseminado de hormônios por indivíduos transexuais sem acompanhamento médico, a alta taxa de manutenção da disforia de gênero na vida adulta, a elevada morbidade, sobretudo psicológica, que se segue ao desenvolvimento de características sexuais opostas ao sexo de identificação, e a disponibilidade de novas drogas para assegurar a segurança e eficácia do tratamento (CFM, 2013).

As implicações das resoluções do CFM são inúmeras, inclusive para que se possa repensar a formação médica, assim como dos profissionais de saúde de um modo geral. Lima e Cerqueira (2008), em estudo que investigou estudantes de Medicina, evidenciaram que uma parte significativa dos alunos consideravam a homossexualidade um comportamento patológico, apesar de a Organização Mundial da Saúde (OMS) ter excluído o "homossexualismo" da lista dos transtornos mentais da Classificação Internacional de Doenças - $10^{a}$ revisão (CID-10) em 1990. Melo (2010) argumenta que a não abordagem de temas relacionados à sexualidade e à diversidade sexual posiciona esses tópicos fora do conhecimento técnico, de tal forma que muitos profissionais passam a considerar que esses assuntos estariam fora do âmbito de seu conhecimento técnico. Em vez disso, entende-se que essas questões são de ordem pessoal e, por isso, estariam fora da competência biomédica (Silva, 2014). O mesmo pode ser dito em relação à identidade de gênero. Sem contar a profunda ignorância que paira sobre esses assuntos, uma vez que a preocupação com o ensino das especificidades da comunidade LGBTT e suas implicações na saúde está praticamente ausente nos currículos das faculdades de Medicina.

Esse panorama da formação médica nos dias de hoje, no que concerne às questões de gênero e sexualidade, oferecem oportunidades exíguas de debate acadêmico a respeito desses temas tão fundamentais para a qualificação das 
profissões de ajuda, como é o caso da carreira médica. Quando se olha para os contextos específicos de expressão de variantes da identidade de gênero, verticalizadas nas transexualidades e nas travestilidades, esse silenciamento que marca a formação médica torna-se ainda mais gritante. É claro que, nesse diapasão, as dificuldades no âmbito do ensino dos construtos teóricos vão se avolumar e reverberar na prática assistencial, quando o encontro com a pessoa em sofrimento físico e psicológico irá despertar inquietações e angústias no futuro médico.

O amplo desconhecimento dos temas relacionados às diversidades de gênero e sexuais constituem entraves para a sensibilização precoce do médico às questões desafiadoras que atravessam a prática profissional. Como conduzir uma consulta ginecológica de uma mulher trans? Como proceder em uma entrevista de anamnese com um homem trans? Como indagar, no exame de saúde mental, qual o impacto que os sintomas (que o paciente descreve) têm em sua vida sexual?

O caráter embaraçoso com que muitas vezes as questões de gênero e sexualidade são abordadas pelo jovem durante o ensino médico evidencia que ainda há um considerável trabalho a ser feito até que haja uma adequada incorporação do reconhecimento dessas dimensões como parte da qualidade de vida do sujeito. Essa transformação requer a adoção de medidas na formação que possam efetivamente contribuir para modelar uma nova mentalidade. Também é preciso tornar o ambiente dos serviços de saúde mais acolhedor para a clientela LGBTT, considerando a vulnerabilidade aumentada pelo fato de estar exposta a inúmeros processos de estimatização.

Entre as medidas necessárias ressalta-se o preparo técnico dos profissionais de saúde para suprir as demandas da população LGBTT, para que se sintam seguros para atender esses indivíduos de forma empática e compreensiva, com uma atitude não patologizante acerca das múltiplas expressões possíveis das identidades de gênero e das orientações sexuais. Isso exige uma mudança de paradigma de formação, além de uma reestruturação do ensino nos cursos de graduação da área de saúde, sobretudo no curso médico, de maneira que temas relacionados à sexualidade humana, orientação sexual, identidade de gênero, homossexualidade, bissexualidade e transexualidade possam ser abordados de maneira consistente e a partir de perspectivas plurais, evitando-se os dogmatismos e reducionismos empobrecedores, como ver a sexualidade e o 
gênero como conceitos intercambiáveis ou restringir a complexidade fascinante dos fenômenos relacionados ao sexo a uma determinação meramente biológica.

É preciso preparar os futuros profissionais de saúde para uma realidade constituída de diversidades de indivíduos únicos e singulares. As pesquisas podem contribuir nessa direção, por exemplo, Silva (2014) investigou os sentidos da diversidade sexual entre estudantes de Medicina. Cardoso e Ferro (2012) estudaram a saúde da população LGBTT, no recorte de suas demandas e especificidades. Cerqueira-Santos et al. (2010) investigaram a percepção de usuários gays, lésbicas, bissexuais e transgêneros (transexuais e travestis) acerca do Sistema Único de Saúde (SUS).

Já existe um corpo de evidências consistentes, que dão sustentação às iniciativas transformadoras da formação e da prática profissional, e que poderão resultar na possibilidade de se assegurar a necessária humanização no acolhimento de gays, lésbicas, bissexuais, travestis e transexuais na atenção básica (Moscheta \& Santos, 2010). A formação do estudante deve se dar em sinergia com a busca da integralidade e da equidade, para que se dê a efetivação dos direitos à saúde e à atenção integral dos LGBTT. É no eixo da formação profissionalizante que se dá o encontro entre a ação técnica e a postura ética nas práticas de saúde. A questão que se coloca é: será possível alavancar uma real transformação da educação médica e formar um "novo médico" apenas a partir de mudanças no método de ensino-aprendizagem (Gomes \& Rego, 2011)?

Colocando o foco especificamente no campo da transexualidade, um dos desafios enfrentados no cenário atual do SUS tem como marca principal a questão de como garantir a atenção integral à saúde no processo transexualizador. Afinal, são muitas as limitações encontradas no cotidiano dos serviços públicos de saúde. Nessa direção, o estudo de Ferraz e Kraiczyk (2010) aproxima gênero das políticas públicas de saúde e discute como construir respostas para o enfrentamento das desigualdades que se verticalizam no âmbito do SUS.

Segundo Ferraz e Kraiczyk (2010), ao longo de duas décadas de consolidação do SUS a dimensão de gênero tem sido incorporada nas políticas públicas de saúde de diversos modos, sendo que a Política Nacional de Saúde Integral de Lésbicas, Gays, Bissexuais, Travestis e Transexuais (Portaria no 457, de 19 de agosto de 2008) é emblemática do modo como isso está se dando. Assim, no âmbito das políticas de saúde, o Ministério da Saúde lançou as bases dessa Política de Atenção Integral à Saúde da População LGBT. Esse documento é 
considerado um dos marcos fundamentais que pontuam os avanços e as mudanças que têm sido implementadas na direção de uma atenção integral à saúde, que assegure de fato a inclusão das diversidades sexuais e de gênero nos cuidados em saúde.

Aprovada pelo Conselho Nacional de Saúde em 2009, mas só efetivamente publicada em 2011 (Popadiuk et al., 2017) e redefinida e ampliada em 2013 (Portaria no 2803, de 19 de novembro de 2013), a Política Nacional de Saúde Integral LGBT representou um passo importante para a busca de maior equidade no SUS. Refletindo essa política, o documento celebra o compromisso do Ministério da Saúde com a redução das desigualdades, constituindo uma das bases do Programa Mais Saúde - Direito de Todos (Brasil, 2008b). Esse programa, lançado em 2008, visava à reorientação das políticas de saúde com objetivo de ampliar o acesso a ações e serviços de qualidade. Para tanto, foram traçadas metas específicas para promover ações de enfrentamento das iniquidades e desigualdades em saúde, especialmente de grupos populacionais historicamente marginalizados como negros, quilombolas, LGBTT, prostitutas, população em situação de rua e ciganos (Portaria no 457, de 19 de agosto de 2008).

Uma das linhas de força da Política Nacional de Saúde Integral LGBT é o reconhecimento explícito dos efeitos da discriminação e exclusão no processo de saúde-doença da população LGBTT (Portaria no 457, de 19 de agosto de 2008). Nesse sentido, as diretrizes e objetivos estabelecidos apontam na direção da promoção de mudanças na matriz de determinação social da saúde, com a pretendida redução das desigualdades relacionadas à saúde dos grupos mais vulneráveis.

Por outro lado, passo a passo com os avanços alcançados, os problemas encontrados para efetivar essa política têm se avolumado nos anos posteriores ao seu lançamento, constituindo impasses que são verdadeiras barreiras ao enfrentamento das desigualdades no âmbito do sistema de saúde (Ferraz \& Kraiczyk, 2010; Lionço, 2009; Popadiuk et al., 2017).

\section{CONSIDERAÇÕES FINAIS}

Desde o primeiro parecer emitido pelo CFP em 1975, que de forma peremptória sentenciava que "o sexo biológico é imutável", a posição oficial da entidade médica no Brasil assumiu de forma inequívoca o entendimento 
referenciado na matriz binária heterossexual para os gêneros. Sexo é biologia; biologia não se muda, se aceita. Essa visão conservadora informou decisões e insuflou polêmicas, como a ameaça de cassação do registro profissional de profissionais que ousassem transgredir os limites estabelecidos pela entidade regulamentadora da profissão médica no país. Esse espírito cerceador continuaria vigente pelas décadas seguintes, ecoando, por exemplo, em outro parecer, emitido pelo CFP em 1991, que evocava um dispositivo do código penal para intimidar os médicos que se atrevessem a realizar o $\operatorname{PrTr}$, alegando "se tratar de mutilação grave e ofensa à integridade corporal".

Identificou-se que o início da mudança da jurisprudência se deu em 1997, portanto, 22 anos após a emissão do primeiro parecer, quando o CFM criou uma comissão de especialistas médicos para estudar o assunto, tendo em vista a preocupação com a repercussão das discussões sobre o assunto nos meios de comunicação. É interessante observar que o documento elaborado por essa comissão considerou que, apesar dos posicionamentos anteriores da entidade representativa da categoria médica serem contrários ao procedimento, o parecer se ampara em "recentes estudos genéticos" que indicavam "bases orgânicas para os desvios de identidade sexual". Ou seja, o suposto progresso do conhecimento médico foi adotado como critério para reformar uma tradição do conselho. As evidências disponíveis na época sugeriam "bases orgânicas" que explicariam as manifestações "desviantes" da identidade sexual, isto é, as expressões que divergem do padrão heteronormativo, até então identificado como o único a ser referenciado na determinação biológica da espécie humana. Contudo, novas descobertas científicas teriam corroborado que também os "desvios de identidade sexual" não escapam da inexorável determinação biológica. Logo, tem-se aí uma base segura para justificar a intervenção sobre a matriz biológica do corpo, com o propósito de corrigir um "erro da natureza".

Outra razão evocada à época para a mudança de posicionamento do Conselho foi "a presença de técnicas seguras e eficazes", que ofereciam segurança na manipulação e correção cirúrgica do "corpo errado". Além disso, em só em terceiro lugar, foram evocadas as "mudanças de costumes e hábitos sociais dos grandes centros urbanos" como justificativa adicional para adotar uma postura liberal em relação à intervenção cirúrgica (CFM, 1997). Desse modo, pela primeira vez houve a manifestação do Conselho francamente favorável à liberação da cirurgia de transgenitalização, ainda que em caráter experimental. 
Uma das lições que podem ser extraídas dessa trajetória histórica sobre a compreensão médica em relação à categoria da transexualidade é o quanto afirmações como "o sexo biológico é imutável" se valem de crenças que supostamente estariam amparadas em conhecimento científico para perpetuarem argumentos morais. Outra conclusão a que se pode chegar facilmente é que a experiência trans, com toda a densidade subjetiva que the é própria e a complexidade das questões médicas, legais, familiares, sociais e religiosas que ela evoca, é sumariamente reduzida, por efeito desse discurso, a uma questão médica.

De acordo com Rocon, Sodré e Rodrigues (2016), o processo transexualizador no contexto brasileiro tem sua história marcada pela medicalização da experiência trans, judicialização da demanda e regulamentação da vida pelo Estado. Segundo esses autores, esse programa, na sua implementação, tem se mostrado altamente seletivo, na medida em que institui como critério de elegibilidade uma conformidade com o que se convencionou como indicação segura para o PrTr. Isso, na prática, permite ao Estado selecionar aqueles que se enquadram nos limites estritos de um diagnóstico referenciado na matriz binária heterossexual para a definição dos gêneros. Desse modo, não se assegura o princípio do acesso universal aos serviços em saúde. Os autores concluem que o PrTr tem servido de dispositivo disciplinador sobre a população trans. Nesse contexto, a seletividade pode ser compreendida como fruto do exercício do biopoder por parte do Estado, que decide quem merece viver ou morrer.

O conhecimento proporcionado por este estudo traz valiosos subsídios para o planejamento e avaliação das políticas públicas na área. Ainda que seja lenta sua implantação, os avanços foram consideráveis. No entanto, atualmente paira um desafio à manutenção e potencialização do PrTr no SUS, com a possibilidade de retrocesso imposta pela política ultraconservadora que tem pautado a atuação do executivo e legislativo no país a partir de janeiro de 2019. Isso aumenta ainda mais a responsabilidade e convoca o espírito de resistência daqueles que acreditam na defesa dos ideais democráticos e lutam por um país com menos desigualdade e mais justiça social.

\section{DECLARAÇÃO DE CONFLITOS DE INTERESSE}

Os autores declaram não haver conflito de interesse. 


\section{REFERÊNCIAS}

Brasil, Ministério da Saúde (2008a). Política Nacional de Saúde Integral de Lésbicas, Gays, Bissexuais, Travestis e Transexuais - LGBT. Brasília, DF: Conselho Nacional de Saúde. Recuperado em 28 de maio de 2019, de http://bvsms.saude.gov.br/bvs/publicacoes/politica nacional saude lesbica s gays.pdf

Brasil. Ministério da Saúde (2008b). Mais saúde: direito de todos: 2008-2011. Brasília, DF: Ministério da Saúde. Recuperado em 28 de maio de 2019, de http://bvsms.saude.gov.br/bvs/publicacoes/mais saude direito todos 3ed. pdf.

Cardoso, M. R., \& Ferro, L. F. (2012). Saúde e população LGBT: demandas e especificidades em questão. Psicologia: Ciência e Profissão, 32(3), 552-563. http://dx.doi.org/10.1590/S1414-98932012000300003.

Cerqueira-Santos, E., Calvetti, P. U., Rocha, K. B., Moura, A., Barbosa, L. H., \& Hermel, J. (2010). Percepção de usuários gays, lésbicas, bissexuais e transgêneros, transexuais e travestis do Sistema Único de Saúde. Revista Interamericana de Psicologia, 44(2), 235-245. Recuperado em 24 de maio de 2019, de http://www.redalyc.org/pdf/284/28420641004.pdf.

Ferraz, D., \& Kraiczyk, J. (2010). Gênero e políticas públicas de saúde: construindo respostas para o enfrentamento das desigualdades no âmbito do SUS. Revista de Psicologia da UNESP, 9(1), 70-82. Recuperado em 24 de maio de 2019, de http://seer.assis.unesp.br/index.php/psicologia/article/view/428.

Gomes, A. P., \& Rego, S. (2011). Transformação da educação médica: é possível formar um novo médico a partir de mudanças no método de ensinoaprendizagem? Revista Brasileira de Educação Médica, 35(4), 557-566. http://dx.doi.org/10.1590/S0100-55022011000400016.

http://dx.doi.org/10.1590/1414-49802016.00200011

Lima, M. C. P., \& Cerqueira, A. T. A. R. (2008). Crenças sobre sexualidade entre estudantes de Medicina: uma comparação entre gêneros. Revista Brasileira de Educação Médica, 32(1), 49-55. http://dx.doi.org/10.1590/S010055022008000100007.

Lionço, T. (2009). Atenção integral à saúde e diversidade no processo transexualizador no SUS: avanços, impasses e desafios. Physis: Revista de Saúde Coletiva, 19(1), 43-63. http://dx.doi.org/10.1590/S010373312009000100004.

Melo, A. P. L. (2010). "Mulher mulher" e outras mulheres": gênero e homossexualidade(s) no Programa de Saúde da Família. Dissertação (Mestrado). Instituto de Medicina Social, Universidade do Estado do Rio de Janeiro, Rio de Janeiro. 
Moscheta, M. S., \& Santos, M. A. (2010). Inclusão e o desafio de criar formas de investigação colaborativa: um relato de experiência. Saúde e Transformação Social, 1(1), 154-159. Recuperado em 24 de maio de 2019, de http://incubadora.periodicos.ufsc.br/index.php/saudeetransformacao/article Lview/415/447.

Popadiuk, G. S., Oliveira, D. C., \& Signorelli, M. C. (2017). A Política Nacional de Saúde Integral de Lésbicas, Gays, Bissexuais e Transgêneros (LGBT) e o acesso ao Processo Transexualizador no Sistema Único de Saúde (SUS): avanços e desafios. Ciência \& Saúde Coletiva, 22(5), 1509-1520. https://dx.doi.org/10.1590/1413-81232017225.32782016

Portaria no 2803, de 19 de novembro de 2013. (2013). Redefine e amplia o Processo Transexualizador no Sistema Único de Saúde (SUS). Diário Oficial da União; 20 novembro 2013.

Portaria no 457, de 19 de agosto de 2008. (2008). Aprova a Regulamentação do Processo Transexualizador no âmbito do Sistema Único de Saúde (SUS). Diário Oficial da União; 20 agosto 2008.

Rocon, P. C., Sodré, F., \& Rodrigues, A. (2016). Regulamentação da vida no processo transexualizador brasileiro: uma análise sobre a política pública. Revista Katálysis, 19(2), 260-269. http://dx.doi.org/10.1590/141449802016.00200011.

Silva, M. C. B. A. (2014). Sentidos da diversidade sexual entre estudantes de Medicina. Dissertação (Mestrado em Ciências Humanas), Universidade Federal de Uberlândia, Uberlândia.

Sobre os autores

Manoel Antônio dos Santos é Professor Titular da Faculdade de Filosofia, Ciências e Letras de Ribeirão Preto da Universidade de São Paulo. É psicólogo, mestre e doutor em Psicologia Clínica pelo Instituto de Psicologia da Universidade de São Paulo (IP-USP). Coordenador do Laboratório de Ensino e Pesquisa em Psicologia da Saúde - LEPPS (FFCLRP-USP-CNPq). Trabalha no campo da Psicologia da Saúde, nas interfaces processo saúde-doença, família, gênero e diversidade sexual. É bolsista de Produtividade em Pesquisa PQ-1A do Conselho Nacional de Desenvolvimento Científico e Tecnológico - CNPq. e-mail:

masantos@ffclrp.usp.br

Ricardo Santos de Souza é acadêmico do curso de medicina da Faculdade de Medicina de Ribeirão Preto da Universidade de São Paulo. Foi bolsista do Programa Unificado de Bolsas de Estudo para Apoio e Formação de Estudantes de Graduação (PUB-USP) de 2016 a 2018. Membro do Laboratório de Ensino e Pesquisa em Psicologia da Saúde - LEPPS (FFCLRP-USP-CNPq). Trabalha no campo da diversidade sexual e identidade de gênero. e-mail:

ricardo dos santos@yahoo.com 
Lúcia Alves da Silva Lara é médica ginecologista e obstetra, Médica Assistente do Setor de Reprodução Humana do Departamento de Ginecologia e Obstetrícia da Faculdade de Medicina de Ribeirão Preto da Universidade de São Paulo (FMRPUSP). Coordenadora do Serviço de Medicina Sexual (Ambulatório de Estudos em Sexualidade Humana - AESH) da FMRP-USP, serviço de referência na assistência à população trans. Trabalha com sexologia, diversidade de orientações sexuais e identidades de gênero. e-mail: luciaalvess@yahoo.com.br Eduardo Name Risk é pós-doutorando da Faculdade de Filosofia, Ciências e Letras de Ribeirão Preto da Universidade de São Paulo (FFCLRP-USP). É psicólogo, mestre e doutor pelo Programa de Pós-Graduação em Psicologia da FFCLRP-USP. Membro do Laboratório de Ensino e Pesquisa em Psicologia da Saúde - LEPPS (FFCLRP-USP-CNPq). Editor Junior da Paidéia (Ribeirão Preto). Bolsista de Pós-Doutoramento pelo Conselho Nacional de Desenvolvimento Científico e Tecnológico (CNPq). Trabalha com família na modernidade, relações de gênero e diversidade sexual. E-mail: eduardonrisk@gmail.com Wanderlei Abadio de Oliveira é Professor Doutor da Escola de Enfermagem de Ribeirão Preto da Universidade de São Paulo (EERP-USP). É psicólogo e doutor pela EERP-USP. Pós-doutorando da Faculdade de Filosofia, Ciências e Letras de Ribeirão Preto da Universidade de São Paulo (FFCLRP-USP). Bolsista do Programa Nacional de Pós-Doutorado PNPD/Capes. Membro do Laboratório de Ensino e Pesquisa em Psicologia da Saúde - LEPPS (FFCLRP-USP-CNPq). Membro da Comissão Editorial da Paidéia (Ribeirão Preto). Trabalha no campo da Psicologia da Saúde e na educação nas interfaces bullying, adolescência, violências e determinantes sociais da saúde. e-mail: wanderleio@usp.br Vinicius Alexandre é psicólogo e mestrando do Programa de Pós-Graduação em Psicologia da Faculdade de Filosofia, Ciências e Letras de Ribeirão Preto da Universidade de São Paulo (FFCLRP-USP). Membro do Laboratório de Ensino e Pesquisa em Psicologia da Saúde - LEPPS (FFCLRP-USP-CNPq). Trabalha no campo da Psicologia da Saúde nas interfaces diversidade de orientações sexuais e identidades de gênero. e-mail: valexandre83@gmail.com Érika Arantes de Oliveira-Cardoso é Professora Doutora do Programa de PósGraduação em Psicologia da Faculdade de Filosofia, Ciências e Letras de Ribeirão Preto da Universidade de São Paulo (FFCLRP-USP). É psicóloga, mestre e doutora pelo Programa de Pós-Graduação em Psicologia da FFCLRP-USP. Especialista em Psicologia Hospitalar pelo Conselho Federal de Psicologia (CFP). Vicecoordenadora do Laboratório de Ensino e Pesquisa em Psicologia da Saúde LEPPS (FFCLRP-USP-CNPq). Trabalha no campo da Psicologia da Saúde, nas interfaces enfrentamento do processo saúde-doença, diversidade de orientações sexuais e identidades de gênero. e-mail: erikaao@ffclrp.usp.br

Certificamos que todos os(as) autores(as) participaram suficientemente do trabalho para tornar pública sua responsabilidade pelo conteúdo. A contribuição 
de cada autor(a) pode ser atribuída como se segue: todos os(as) autores(as) foram responsáveis pela conceitualização, investigação e visualização do artigo e trabalharam na redação inicial do texto (revisão e edição).

Os autores agradecem ao Ambulatório de Estudos da Sexualidade Humana (AESH) do Hospital das Clínicas da Faculdade de Medicina de Ribeirão Preto da Universidade de São Paulo, pelo apoio à pesquisa e formação em serviço de profissionais de diferentes áreas.

Apoio: bolsa de Produtividade em Pesquisa PQ-1A do Conselho Nacional de Desenvolvimento Científico e Tecnológico - CNPq.

Recebido em: 06/05/2019 Aceito em: 28/05/2019 